\title{
Stability in Switched Cohen-Grossberg Neural Networks with Mixed Time Delays and Non-Lipschitz Activation Functions
}

\author{
Huaiqin Wu, ${ }^{1}$ Guohua $X u^{1}{ }^{1}$ Chongyang $W u^{2}{ }^{2} N^{2}$ Li, ${ }^{1}$ \\ Kewang Wang, ${ }^{1}$ and Qiangqiang Guo ${ }^{1}$ \\ ${ }^{1}$ College of Science, Yanshan University, Qinhuangdao 066001, China \\ ${ }^{2}$ College of Economics and Finance, Huaqiao University, Quanzhou 362021, China
}

Correspondence should be addressed to Huaiqin Wu, huaiqinwu@ysu.edu.cn

Received 20 January 2012; Accepted 25 March 2012

Academic Editor: Piyapong Niamsup

Copyright (c) 2012 Huaiqin Wu et al. This is an open access article distributed under the Creative Commons Attribution License, which permits unrestricted use, distribution, and reproduction in any medium, provided the original work is properly cited.

The stability for the switched Cohen-Grossberg neural networks with mixed time delays and $\alpha$ inverse Hölder activation functions is investigated under the switching rule with the average dwell time property. By applying multiple Lyapunov-Krasovskii functional approach and linear matrix inequality (LMI) technique, a delay-dependent sufficient criterion is achieved to ensure such switched neural networks to be globally exponentially stable in terms of LMIs, and the exponential decay estimation is explicitly developed for the states too. Two illustrative examples are given to demonstrate the validity of the theoretical results.

\section{Introduction}

In the past few decades, there has been increasing interest in different classes of neural networks such as Hopfield, Cellular, Cohen-Grossberg, and bidirectional associative neural networks due to their potential applications in many areas such as classification, signal and image processing, parallel computing, associate memories, optimization, and cryptography [1-5]. In the design of practical neural networks, the qualitative analysis of neural network dynamics plays an important role. To solve problems of optimization, neural control, and signal processing, neural networks have to be designed in such a way that, for a given external input, they exhibit only one globally asymptotically/exponentially stable equilibrium point. Hence, much effort has been made in the stability of neural networks, and a number of sufficient conditions have been proposed to guarantee the global asymptotic/exponential stability for neural networks with or without delays in recent years, see, for example, [6-26] and the references therein. 
Recently, by combing the theories of the switched systems and neural networks, several classes of mathematics models of switched neural networks have been established. As a special class of switched systems, switched neural networks, whose individual subsystems are a set of neural networks, have found applications in fields of high speed signal processing, artificial intelligence, and gene selection in a DNA microarray analysis [27-30].

Stability issues of switched neural networks have received great attention of researchers so far [31-39]. In [31], based on the Lyapunov-Krasovskii method and LMI approach, some sufficient conditions were derived for global robust exponential stability of a class of switched Hopfield neural networks with time-varying delay under uncertainty. In [32], by combining Cohen-Grossberg neural networks with an arbitrary switching rule, the mathematical model of a class of switched Cohen-Grossberg neural networks with mixed time varying delays was established, and the robust stability for such switched CohenGrossberg neural networks was analyzed. In [33], by employing nonlinear measure and LMI techniques, some new sufficient conditions were obtained to ensure global robust asymptotical stability and global robust exponential stability of the unique equilibrium for a class of switched recurrent neural networks with time-varying delay. In [34], authors investigated a large class of switched recurrent neural networks with time-varying structured uncertainties and time-varying delay, some delay-dependent robust periodicity criteria guaranteeing the existence, uniqueness, and global asymptotic stability of periodic solution for all admissible parametric uncertainties were devised by employing free weighting matrices and LMIs. In [35], a new class of switched interval neural networks with discrete and distributed time-varying delays of neural type was developed, and a delay-dependent sufficient criterion was also obtained in terms of LMIs which guarantee the global robust exponential stability for the proposed switched interval neural networks. It should be pointed out that results in [31-35] focused on the stability of switched neural networks under arbitrary switching rule by using common Lyapunov function method. However, common Lyapunov function method requires all the subsystems of the switched system to share a positive definite radially unbounded common Lyapunov function. Generally, this requirement is difficult to achieve.

In past few years, much attention has been paid to making use of the dwell time approach to deal with the analysis and synthesis of switched neural networks [36-39]. It should be pointed out that the average dwell time method is regarded as an important and attractive method to find a suitable switching signal to guarantee switched system stability or improve other performance and has been widely applied to investigate the analysis and synthesis for switched system with or without time-delay, see for example, [40-44]. Very recently, in [36], based on multiple Lyapunov functions method and LMI techniques, the authors presented some sufficient conditions in terms of LMIs which guarantee the robust exponential stability for uncertain switched Cohen-Grossberg neural networks with interval time-varying delay and distributed time-varying delay under the switching rule with the average dwell time property. In [37], by using the average dwell time method, the delaydependent sufficient conditions were derived towards the robust exponential stability for a class of discrete-time switched Hopfield neural networks with time delay. In [38], by applying a new Lyapunov-Krasovskii functional and the average dwell time method, a delay-rangedependent exponential stability criteria and decay estimation are presented in terms of LMIs for switched Hopfield neural networks.

It should be noted that, all the results reported in [31-39] are concerned with switched neural networks with Lipschitz activation functions. To the best of our knowledge, very 
little attention has been paid to the problem of delay-dependent stability for switched neural networks without Lipschitz activation functions, which motivates the work of this paper.

In this paper, our aim is to study the delay-dependent exponential stability problem for a class of switched Cohen-Grossberg neural networks with mixed time delays and $\alpha$ inverse Hölder activation functions. Here, it should be pointed out that $\alpha$-inverse Hölder activation functions are a class of non-Lipschitz functions. By applying Brouwer degree properties, LMI technique and constructing a novel Lyapunov-Krasovskii functional, the existence, uniqueness and global exponential stability of equilibrium point are proved for Cohen-Grossberg neural networks with mixed time delays and $\alpha$-inverse Hölder activation functions. By means of the multiple Lyapunov-Krasovskii functional and the average dwell time approach, a delay-dependent sufficient condition in terms of LMIs is presented to ensure to the considered switched neural networks to be globally exponentially stable, and a explicit expression for the exponential decay estimation is also obtained for the states. Two illustrative examples are given to demonstrate the validity of the theoretical results.

The rest of this paper is organized as follows. In Section 2, the model formulation and some preliminaries are given. In Section 3, the existence, uniqueness, and global exponential stability of equilibrium point are proved for Cohen-Grossberg neural networks with mixed time delays and $\alpha$-inverse Hölder activation functions. In Section 4 , the global exponential stability criterion and state decay estimation are presented for the switched Cohen-Grossberg neural networks with mixed time delays and $\alpha$-inverse Hölder activation functions. In Section 5, two numerical examples are presented to demonstrate the validity of the proposed results. Some conclusions are made in Section 6.

Notations. Throughout this paper, $R$ denotes the set of real numbers, $R^{n}$ denotes the $n$ dimensional Euclidean space, $R^{m \times n}$ denotes the set of all $m \times n$ real matrices. For any matrix $A, A^{T}$ denotes the transpose of $A . A^{-1}$ denotes the inverse of $A$. If $A$ is a real symmetric matrix, $A>0(A<0)$ means that $A$ is positive definite (negative definite). Given the column vectors $x=\left(x_{1}, \ldots, x_{n}\right)^{T}, y=\left(y_{1}, \ldots, y_{n}\right)^{T} \in R^{n}, x^{T} y=\sum_{i=1}^{n} x_{i} y_{i} \cdot\|x\|=\left(\sum_{i=1}^{n} x_{i}^{2}\right)^{1 / 2} \cdot \dot{x}(t)$ denotes the derivative of $x(t), *$ represents the symmetric form of matrix.

\section{Neural Network Model and Preliminaries}

The Cohen-Grossberg neural networks with mixed time delays can be described by the following differential equation system

$$
\dot{x}(t)=\alpha(x(t))\left[-\beta(x(t))+W_{0} g(x(t))+W_{1} g(x(t-\tau))+W_{2} \int_{t-\tau}^{t} g(x(s)) d s+J\right],
$$

where $x(t)=\left(x_{1}(t), \ldots, x_{n}(t)\right)^{T}$ is the vector of neuron states at time $t ; \alpha(x(t))=$ $\operatorname{diag}\left(\alpha_{1}\left(x_{1}(t)\right), \ldots, \alpha_{n}\left(x_{n}(t)\right)\right)$ represents the amplification function; $\beta(x(t))=$ $\operatorname{diag}\left(\beta_{1}\left(x_{1}(t)\right), \ldots, \beta_{n}\left(x_{n}(t)\right)\right)$ is the behaved function; $g(x(t))=\left(g_{1}\left(x_{1}(t)\right), \ldots, g_{n}\left(x_{n}(t)\right)\right)^{T}$ is called the neuron activation function; $W_{i}, i=0,1,2$, are the connection weight matrices; $\tau>0$ denotes the discrete and distributed time delay; $J=\left(J_{1}, \ldots, J_{n}\right)^{T}$ denotes the external input. The initial value associated with (2.1) is assumed to be $x(s)=\varphi(s)$, and $\varphi(s)$ is a continuous function on $[-\tau, 0]$.

In the following, some definitions and lemmas, which play important roles in the proof of our theorems below, are introduced. 
Definition 2.1. The equilibrium point $x^{*}$ of the neural networks (2.1) is said to be globally exponentially stable, if there exist scalars $\eta>0, \tau>0$, and $\delta>0$, such that

$$
\left\|x(t)-x^{*}\right\| \leq \eta e^{-\delta t}, \quad t \geq \tau,
$$

where $x(t)$ is the solution of the system (2.1) with the initial value $x(s)=\varphi(s)$, and $s \in[-\tau, 0]$, $\delta$ is called the exponential convergence rate.

Definition 2.2 (see $[23,24]$ ). A continuous function $\mathcal{G}: R \rightarrow R$,is said to be an $\alpha$-inverse Hölder function, if

(i) $\mathcal{G}$ is a monotonic nondecreasing function,

(ii) for any $\rho \in R$, there exist constants $q_{\rho}>0$ and $r_{\rho}>0$ which are correlated with $\rho$, satisfying

$$
|\mathcal{G}(\theta)-\mathcal{G}(\rho)| \geq q_{\rho}|\theta-\rho|^{\alpha}, \quad \forall|\theta-\rho| \leq r_{\rho}
$$

where $\alpha>0$ is a constant.

The class of $\alpha$-inverse Hölder functions is denoted by $\supset \mathscr{\ell}(\alpha)$. There are a great number of functions which belong to $\supset \mathscr{H}(\alpha)$. For example, $\mathcal{G}(\theta)=\arctan \theta, g(\theta)=\theta^{3}+\theta \in \supset \mathscr{\ell}(1)$, and $\mathcal{G}(\theta)=\theta^{3} \in \supset \mathscr{H}(3)$.

Remark 2.3. If a continuous function $\mathcal{G}: R \rightarrow R$ satisfies that

$$
|\mathcal{G}(\theta)-\mathcal{G}(\rho)| \leq \ell|\theta-\rho|, \quad \forall \theta, \rho \in R
$$

where $\ell>0$ is a constant, then $\mathcal{G}$ is said to be a Lipschitz-continuous function. When $\alpha=1$ and $q_{\rho}$ is independent on $\rho, 1$-inverse Hölder functions are called inverse Lipschitz functions. It is easy to see that $\alpha$-inverse Hölder functions are a class of non-Lipschitz functions.

Let function $\mathscr{F}: R^{n} \rightarrow R^{n}$ be locally Lipschitz continuous. According to Rademacher's theorem [45], $\mp$ is differentiable almost everywhere. Let $D_{\mp}$ denote the set of those points where $\mathcal{F}$ is differentiable, then, $\dot{\mathcal{F}}(x)$ is the Jacobian of $\mathcal{F}$ at $x \in D_{\mathcal{F}}$ and the set $D_{\mathcal{F}}$ is dense in $R^{n}$. The generalized Jacobian of a locally Lipschitz function is defined as follows.

Definition 2.4. For any $x \in R^{n}$, the generalized Jacobian $\partial \mathcal{F}(x)$ of a locally Lipschitz continuous function $\mathcal{F}: R^{n} \rightarrow R^{n}$ is a set of matrices defined by

$$
\partial \mathcal{F}(x)=\operatorname{co}\left\{\mathcal{W} \mid \text { there exists a sequence }\left\{x^{k}\right\} \subset D_{\mathcal{F}} \text { with } \lim _{x^{k} \rightarrow x} \dot{\mathcal{F}}\left(x^{k}\right)=\mathcal{W}\right\},
$$

where $\operatorname{co}(\cdot)$ denotes the convex hull of a set.

The generalized Jacobian is a natural generalization of the Jacobian for continuously differentiable functions, at those points $x$, where $\mathcal{F}$ is continuously differentiable, $\partial \mathcal{F}(x)$ reduces to a single matrix which is Jacobian $\dot{\ddagger}(x)$ of $\mathscr{F}$. 
Definition 2.5. For any switching signal $\sigma(t)$ and any finite constants $T_{1}, T_{2}$ satisfying $T_{2}>$ $T_{1} \geq 0$, let $N_{\sigma}\left(T_{1}, T_{2}\right)$ denote the switching times on the time interval $\left(T_{1}, T_{2}\right)$. If $N_{\sigma}\left(T_{1}, T_{2}\right) \leq$ $N_{0}+\left(T_{2}-T_{1}\right) / T_{a}$ holds for $T_{a}>0, N_{0} \geq 0$, then $T_{a}$ is said to be the average dwell time.

Lemma 2.6 (see $[23,24])$. If $\mathcal{G}(\theta) \in \supset \mathscr{H}(\alpha)$, then for any $\rho_{0} \in R$, one has

$$
\int_{\rho_{0}}^{+\infty}\left[\mathcal{G}(\theta)-\mathcal{G}\left(\rho_{0}\right)\right] d \theta=\int_{\rho_{0}}^{-\infty}\left[\mathcal{G}(\theta)-\mathcal{G}\left(\rho_{0}\right)\right] d \theta=+\infty
$$

Lemma 2.7 (see $[23,24]$ ). If $\mathcal{G}(\theta) \in \supset \mathscr{\ell}(\alpha)$ and $\mathcal{G}(0)=0$, then there exist constants $q_{0}>0$ and $r_{0}>0$, such that

$$
|\mathcal{G}(\theta)| \geq q_{0}|\theta|^{\alpha}, \quad \forall|\theta| \leq r_{0} .
$$

Moreover,

$$
|\mathcal{G}(\theta)| \geq q_{0} r_{0}^{\alpha}, \quad \forall|\theta| \geq r_{0}
$$

Lemma 2.8 (see [25]). Let $\mathcal{F}: R^{n} \rightarrow R^{n}$ be locally Lipschitz continuous. For any given $x, y \in R^{n}$, there exists an element $\mathcal{W}$ in the union $\cup_{z \in[x, y] \partial \mp(z)}$ such that

$$
\mp(y)-F(x)=\mathcal{W}(y-x)
$$

where $[x, y]$ denotes the segment connecting $x$ and $y$.

Let $\Omega$ be a nonempty, bounded, and open subset of $R^{n}$. The closure and boundary of $\Omega$ are denoted by $\bar{\Omega}$ and $\partial \Omega$, respectively.

Lemma 2.9 (see [46]). (1) Let $\mathscr{H}:[0,1] \times \bar{\Omega} \rightarrow R^{n}$ be a continuous mapping. If $p \bar{\epsilon} \mathscr{H}(\lambda, \partial \Omega)$ for all $\lambda \in[0,1]$, then Brouwer degree $\operatorname{deg}(\mathscr{H}(\lambda, \cdot), \Omega, p)$ is constant $(\forall \lambda \in[0,1])$. In this case, one has $\operatorname{deg}(\mathscr{H}(0, \cdot), \Omega, p)=\operatorname{deg}(\mathscr{H}(1, \cdot), \Omega, p)$.

(2) Let $\mathfrak{H}: \bar{\Omega} \rightarrow R^{n}$ be a continuous mapping. If $\operatorname{deg}(\mathfrak{H}, \Omega, p) \neq 0$, then the equation $\mathfrak{H}(x)=p$ has at least a solution in $\Omega$.

Lemma 2.10. Let $x, y \in R^{n}$ and $G>0$, then

$$
2 x^{T} y \leq x^{T} G x+y^{T} G^{-1} y .
$$

Lemma 2.11 (Schur complement). Given constant symmetric matrices $\Sigma_{1}, \Sigma_{2}, \Sigma_{3}$ where $\Sigma_{1}=\Sigma_{1}^{T}$ and $0<\Sigma_{2}=\Sigma_{2}^{T}$, then $\Sigma_{1}+\Sigma_{3}^{T} \Sigma_{2}^{-1} \Sigma_{3}<0$ if and only if

$$
\left[\begin{array}{cc}
\Sigma_{1} & \Sigma_{3}^{T} \\
\Sigma_{3} & -\Sigma_{2}
\end{array}\right]<0, \quad \text { or } \quad\left[\begin{array}{cc}
-\Sigma_{2} & \Sigma_{3}^{T} \\
\Sigma_{3} & \Sigma_{1}
\end{array}\right]<0
$$


Lemma 2.12 (Jensen's inequality). For any constant matrix $\Omega \in R^{n \times n}$, and $\Omega=\Omega^{T}>$ 0 , scalar $\gamma>0$, vector function $\omega:[0, \gamma] \rightarrow R^{n}$, such that the integrations concerned are well defined, then

$$
\frac{1}{\gamma}\left(\int_{0}^{\gamma} \omega(s) d s\right)^{T} \Omega\left(\int_{0}^{\gamma} \omega(s) d s\right) \leq \int_{0}^{\gamma} \omega(s)^{T} \Omega \omega(s) d s .
$$

To give our main results in the next sections, we need to present the following assumptions.

$\left(H_{1}\right) \alpha_{i}(s)$ is continuous, and $0<\underline{\alpha}_{i}<\alpha_{i}(s)<\bar{\alpha}_{i}$ for all $s \in R, i=1,2, \ldots, n$.

$\left(H_{2}\right) \beta_{i}(s)$ is locally Lipschitz continuous, and there exists a constant $\beta_{i}>0$ such that $\dot{\beta}_{i}(s) \geq \beta_{i}$ for all $s \in R$ at which $\beta_{i}(s)$ is differentiable, $i=1,2, \ldots, n$.

$\left(H_{3}\right) g_{i}(s) \in \supset \mathscr{H}(\alpha), i=1,2, \ldots, n$.

\section{Exponential Stability of the Cohen-Grossberg Neural Network}

In this section, the Cohen-Grossberg neural network (2.1) is considered the main results on the existence and stability of equilibrium point of the neural network (2.1) will be presented in the following theorem.

Theorem 3.1. Under the assumptions $\left(H_{1}\right)--\left(H_{3}\right)$, if there exist two positive definite matrices $S, T$, two positive definite diagonal matrices $M, P$ and a scalar $\gamma>0$ such that

$$
\left[\begin{array}{cccc}
\gamma P \underline{A}^{-1}-P B-B P & P W_{0}+\gamma M A^{-1}-M B & P W_{1} & P W_{2} \\
W_{0}^{T} P+\gamma \underline{A}^{-1} M-B M & M W_{0}+W_{0}^{T} M+S+\tau T & M W_{1} & M W_{2} \\
W_{1}^{T} P & W_{1}^{T} M & -e^{-\gamma \tau} S & 0 \\
W_{2}^{T} P & W_{2}^{T} M & 0 & -\frac{e^{-\gamma \tau}}{\tau} T
\end{array}\right]<0
$$

is satisfied, where $\underline{A}=\operatorname{diag}\left(\underline{\alpha}_{1}, \underline{\alpha}_{2}, \ldots, \underline{\alpha}_{n}\right), B=\operatorname{diag}\left(\beta_{1}, \beta_{2}, \ldots, \beta_{n}\right), M=\operatorname{diag}\left(m_{1}, m_{2}, \ldots, m_{n}\right)$, $P=\operatorname{diag}\left(p_{1}, p_{2}, \ldots, p_{n}\right)$, then the Cohen-Grossberg neural network (2.1) has one unique equilibrium point which is globally exponentially stable.

Proof. We should prove this theorem in three steps.

Step 1. In this step, we will prove the existence of the equilibrium point.

Let $\mathfrak{H}(x)=\beta(x)-\left(W_{0}+W_{1}+\tau W_{2}\right) g(x)-J$. By the assumption $\left(H_{1}\right), x^{*} \in R^{n}$ is an equilibrium point of the system (2.1) if and only if $\mathfrak{H}\left(x^{*}\right)=0$. Rewrite $\mathfrak{H}(x)$ as

$$
\mathfrak{H}(x)=\tilde{\beta}(x)-\left(W_{0}+W_{1}+\tau W_{2}\right) \tilde{g}(x)+\mathfrak{H}(0),
$$

where $\tilde{\beta}(x)=\beta(x)-\beta(0), \quad \tilde{g}(x)=g(x)-g(0)$. Obviously, $\widetilde{g}_{i}(0)=0, \tilde{g}_{i} \in \supset \mathscr{L}(\alpha)$ and $x_{i} \widetilde{g}_{i}\left(x_{i}\right)>$ $0\left(x_{i} \neq 0\right)$. By Lemma 2.8 and the assumption $\left(H_{2}\right)$, it can follow that $\tilde{\beta}(x)=\beta(x)-\beta(0)=$ $\widetilde{B} x, \widetilde{B} \in \cup_{z \in[0, x]} \partial \beta(z)$, where $\widetilde{B}=\operatorname{diag}\left(\widetilde{\beta}_{1}, \widetilde{\beta}_{2}, \ldots, \widetilde{\beta}_{n}\right)$ with $\widetilde{\beta}_{i} \geq \beta_{i}, i=1,2, \ldots, n$. 
Discrete Dynamics in Nature and Society

Let $\Omega_{\mathcal{R}}=\left\{x \in R^{n}:\|x\|<\mathcal{R}\right\}, \quad \mathcal{R}>0$. Define the mapping $\mathscr{H}:[0,1] \times \bar{\Omega} \rightarrow R^{n}$ as

$$
\mathscr{H}(\lambda, x)=\widetilde{B} x-\lambda\left(W_{0}+W_{1}+\tau W_{2}\right) \tilde{g}(x)+\lambda \mathfrak{H}(0),
$$

where $\bar{\Omega}_{\mathcal{R}}=\left\{x \in R^{n}:\|x\| \leq \mathcal{R}\right\}$.

From (3.1) and the definition of negative definite matrix, we can obtain

$$
\left[\begin{array}{ccc}
M W_{0}+W_{0}^{T} M+S+\tau T & M W_{1} & M W_{2} \\
W_{1}^{T} M & -\mathrm{e}^{-\gamma \tau} S & 0 \\
W_{2}^{T} M & 0 & -\frac{\mathrm{e}^{-\gamma \tau}}{\tau} T
\end{array}\right]<0
$$

By Lemma 2.11, (3.4) is equivalent to

$$
M W_{0}+W_{0}^{T} M+S+\mathrm{e}^{r \tau} M W_{1} S^{-1} W_{1}^{T} M+\tau \mathrm{e}^{\gamma \tau} M W_{2} T^{-1} W_{2}^{T} M+\tau T<0 .
$$

By means of Lemma 2.10, we have

$$
\begin{aligned}
& \tilde{g}(x)^{T} M W_{1} \tilde{g}(x) \leq \frac{1}{2}\left[\tilde{g}(x)^{T} M W_{1} S^{-1} W_{1}^{T} M \widetilde{g}(x)+\tilde{g}(x)^{T} S \tilde{g}(x)\right] \\
& \tilde{g}(x)^{T} M W_{2} \tilde{g}(x) \leq \frac{1}{2}\left[\tilde{g}(x)^{T} M W_{2} T^{-1} W_{2}^{T} M \widetilde{g}(x)+\tilde{g}(x)^{T} T \widetilde{g}(x)\right] .
\end{aligned}
$$

By using (3.5) and (3.6), we have

$$
\begin{aligned}
& \tilde{g}(x)^{T} M \mathscr{\ell}(\lambda, x) \\
& =\tilde{g}(x)^{T} M \widetilde{B} x+\lambda \widetilde{g}(x)^{T} M \mathfrak{H}(0)-\lambda \widetilde{g}(x)^{T} M\left(W_{0}+W_{1}+\tau W_{2}\right) \tilde{g}(x) \\
& =\tilde{g}(x)^{T} M(\widetilde{B} x+\lambda \mathfrak{H}(0))-\lambda \tilde{g}(x)^{T} M\left(W_{0}+W_{1}+\tau W_{2}\right) \tilde{g}(x) \\
& \geq \widetilde{g}(x)^{T} M(\widetilde{B} x+\lambda \mathfrak{H}(0))-\frac{\lambda}{2}\left[\widetilde { g } ( x ) ^ { T } \left(M W_{0}+W_{0}^{T} M+S+M W_{1} S^{-1} W_{1}^{T} M\right.\right. \\
& \left.\left.+\tau M W_{2} T^{-1} W_{2}^{T} M+\tau T\right) \tilde{g}(x)\right] \\
& \geq \tilde{g}(x)^{T} M(\widetilde{B} x+\lambda \mathfrak{H}(0)) \\
& \geq \sum_{i=1}^{n}\left[m_{i} \tilde{\beta}_{i}\left|\widetilde{g}_{i}\left(x_{i}\right)\right|\left|x_{i}\right|-\left|\tilde{g}_{i}\left(x_{i}\right)\right|\left|(M \mathfrak{H}(0))_{i}\right|\right] \\
& =\sum_{i=1}^{n} m_{i} \tilde{\beta}_{i}\left|\tilde{g}_{i}\left(x_{i}\right)\right|\left[\left|x_{i}\right|-\frac{\left|(M \mathfrak{H}(0))_{i}\right|}{m_{i} \tilde{\beta}_{i}}\right] \text {, }
\end{aligned}
$$

where $(M \mathfrak{H}(0))_{i}(i=1,2, \ldots, n)$ denotes the $i$ th element of vector $(M \mathfrak{H}(0))$.

By virtue of Lemma 2.7, there exist constants $q_{i_{0}}>0$ and $r_{i_{0}}>0, i=1,2, \ldots, n$, such that

$$
\left|\tilde{g}_{i}\left(x_{i}\right)\right| \geq q_{i_{0}} r_{i_{0}}^{\alpha}, \quad \forall\left|x_{i}\right| \geq r_{i_{0}}, \quad i=1,2, \ldots, n
$$


Let $a=\max _{1 \leq i \leq n}\left(\left|(M \mathfrak{H}(0))_{i}\right| / m_{i} \tilde{\beta}_{i}\right), N_{k}=\left\{n_{1}, \ldots, n_{k}\right\} \subset\{1,2, \ldots, n\}$, forall $k<n$. Define $\Omega_{\mathcal{N}_{k}}=\left\{x:\left|x_{i}\right| \leq a, i \in \mathcal{N}_{k}, x \in R^{k}\right\}, \tilde{g}_{\mathcal{N}_{k}}(x)=\sum_{i \in \mathcal{N}_{k}} m_{i} \tilde{\beta}_{i}\left|\tilde{g}_{i}\left(x_{i}\right)\right|\left[\left|x_{i}\right|-a\right]$. Noting that $\Omega_{N_{k}}$ is a compact subset of $R^{k}$, and $\widetilde{g}_{N_{k}}$ is continuous on $\Omega_{\mathcal{N}_{k}}$, it follows that $\tilde{g}_{N_{k}}$ can reach its the minimum $\min _{x \in \Omega_{\mathcal{N}_{k}}} \tilde{g}_{\mathcal{N}_{k}}(x)$ on $\Omega_{\mathcal{N}_{k}}$.

Let $r_{0}=\max _{1 \leq i \leq n} r_{i_{0}}, l=\min _{1 \leq i \leq n}\left\{m_{i} \tilde{\beta}_{i} q_{i_{0}} r_{i_{0}}^{\alpha}\right\}, \mathcal{M}_{\mathcal{N}_{k}}=\min _{x \in \Omega_{\mathcal{N}_{k}}} \tilde{g}_{\mathcal{N}_{k}}(x)$, and $\boldsymbol{M}=$ $\min \left\{\mathcal{M}_{\mathcal{N}_{k}}: \mathcal{N}_{k} \subset\{1,2, \ldots, n\}\right\}$. Set $\mathcal{R}>\max \left\{\sqrt{n}(a-\mathcal{M} / l), \sqrt{n} r_{0}\right\}$ and $x \in \partial \Omega_{R}$, then there exist two index sets $\mathcal{N}$ and $\overline{\mathcal{N}}$, such that

$$
\left|x_{i}\right| \leq a, \quad i \in \mathcal{N}, \quad\left|x_{i}\right|>a, \quad i \in \overline{\mathcal{N}}
$$

where $\mathcal{N} \overline{\mathcal{N}}=\{1,2, \ldots, n\}$. Furthermore, there exists an index $\bar{i}_{0}$ in $\overline{\mathcal{N}}$ such that

$$
\left|x_{\overline{i_{0}}}\right| \geq \frac{\mathcal{R}}{\sqrt{n}} \geq \max \left\{a, r_{0}\right\}
$$

By (3.8) and (3.10), for any $x \in \partial \Omega_{R}$ and $\lambda \in[0,1]$,

$$
\begin{aligned}
& (\widetilde{g}(x))^{T} \operatorname{Md\ell }(\lambda, x) \geq \sum_{i \in \mathcal{N}} m_{i} \tilde{\beta}_{i}\left|\widetilde{g}_{i}\left(x_{i}\right)\right|\left[\left|x_{i}\right|-a\right]+\sum_{i \in \bar{N}} m_{i} \tilde{\beta}_{i}\left|\widetilde{g}_{i}\left(x_{i}\right)\right|\left[\left|x_{i}\right|-a\right] \\
& \geq \mathcal{M}+m_{\overline{i_{0}}} \tilde{\beta}_{\bar{i}_{0}}\left|\tilde{g}_{\bar{i}_{0}}\left(x_{\bar{i}_{0}}\right)\right|\left[\left|x_{\bar{i}_{0}}\right|-a\right] \\
& \geq \mathcal{M}+m_{\overline{i_{0}}} \tilde{\beta}_{\bar{i}_{0}} q_{\bar{i}_{0}} r_{\bar{i}_{0}}^{\alpha}\left[\left|x_{\bar{i}_{0}}\right|-a\right] \\
& \geq m_{\bar{i}_{0}} \tilde{\beta}_{\bar{i}_{0}} q_{\bar{i}_{0}} r_{\bar{i}_{0}}^{\alpha}\left[\left|x_{\bar{i}_{0}}\right|-a+\frac{M}{l}\right] \\
& \geq m_{\bar{i}_{0}} \widetilde{\beta}_{\bar{i}_{0}} q_{\bar{i}_{0}} r_{\bar{i}_{0}}^{\alpha}\left[\frac{R}{\sqrt{n}}-a+\frac{M}{l}\right] \\
& >0 \text {. }
\end{aligned}
$$

Hence, this implies that $\mathscr{\ell}(\lambda, x) \neq 0$ for any $x \in \partial \Omega_{R}$ and $\lambda \in[0,1]$. By applying Lemma 2.9 (1), it follows

$$
\operatorname{deg}\left(\mathscr{L}(0, x), \Omega_{\mathcal{R}}, 0\right)=\operatorname{deg}\left(\mathscr{\ell}(1, x), \Omega_{\mathcal{R}}, 0\right),
$$

that is, $\operatorname{deg}\left(\mathfrak{H}(x), \Omega_{\mathcal{R}}, 0\right)=\operatorname{deg}\left(\widetilde{B} x, \Omega_{\mathcal{R}}, 0\right)=\operatorname{sgn}|\widetilde{B}| \neq 0$, where $|\widetilde{B}|$ is the determinant of $\widetilde{B}$. By Lemma $2.9(2), \mathfrak{H}(x)=0$ has at least one solution in $\Omega_{\mathcal{R}}$, that is, the system (2.1) has at least an equilibrium point.

Step 2. In this step, the proof of the uniqueness of the equilibrium point by the method of contradiction will be given.

Assume that $x_{1}^{*}$ and $x_{2}^{*}$ are two different equilibrium points of the system (2.1), then

$$
\beta\left(x_{1}^{*}\right)-\beta\left(x_{2}^{*}\right)=\left(W_{0}+W_{1}+\tau W_{2}\right)\left[g\left(x_{1}^{*}\right)-g\left(x_{2}^{*}\right)\right] .
$$


From Lemma 2.8, it can follow that

$$
\beta\left(x_{1}^{*}\right)-\beta\left(x_{2}^{*}\right)=\widehat{B}\left(x_{1}^{*}-x_{2}^{*}\right),
$$

where $\widehat{B} \in \cup_{z \in\left[x_{1}^{*}, x_{2}^{*}\right]} \partial \beta(z), \widehat{B}=\operatorname{diag}\left(\widehat{\beta}_{1}, \widehat{\beta}_{2}, \ldots, \widehat{\beta}_{n}\right)$ with $\widehat{\beta}_{i} \geq \beta_{i}, i=1,2, \ldots, n$.

By means of (3.5), (3.6), (3.13), and (3.14), it follows that

$$
\begin{aligned}
0< & {\left[g\left(x_{1}^{*}\right)-g\left(x_{2}^{*}\right)\right]^{T} M \widehat{B}\left(x_{1}^{*}-x_{2}^{*}\right) } \\
= & {\left[g\left(x_{1}^{*}\right)-g\left(x_{2}^{*}\right)\right]^{T} M\left(W_{0}+W_{1}+\tau W_{2}\right)\left[g\left(x_{1}^{*}\right)-g\left(x_{2}^{*}\right)\right] } \\
\leq & \frac{1}{2}\left[g\left(x_{1}^{*}\right)-g\left(x_{2}^{*}\right)\right]^{T} \\
& \times\left(M W_{0}+W_{0}^{T} M+S+M W_{1} S^{-1} W_{1}^{T} M+\tau M W_{2} T^{-1} W_{2}^{T} M+\tau T\right)\left[g\left(x_{1}^{*}\right)-g\left(x_{2}^{*}\right)\right] \\
< & 0 .
\end{aligned}
$$

This is a contradiction. Hence, $x_{1}^{*}=x_{2}^{*}$. This shows that the equilibrium point of the system (2.1) is unique.

Step 3. In this step, we will prove that the system (2.1) is globally exponentially stable.

Let $F(x, t)=\alpha(x)\left[-\beta(x)+W_{0} g(x)+W_{1} g\left(x_{\tau}\right)+W_{2} \int_{t-\tau}^{t} g(x(s)) d s+J\right]$, where $x_{\tau}(t)=$ $x(t-\tau)$. Since $g_{i} \in \supset \mathscr{H}(\alpha), \alpha(x), \beta(x)$ are continuous functions, $F(x, t)$ are continuous and locally bounded. Hence, we can obtain the existence of the local solution of the system (2.1) with initial value $x(t)=\varphi(t), t \in[-\tau, 0]$ on $\left[0, t^{*}(\varphi)\right)$, where $t^{*}(\varphi) \in(0,+\infty)$ or $t^{*}(\varphi)=+\infty$, and $\left[0, t^{*}(\varphi)\right)$ is the maximal right-side existence interval of the local solution.

Let $x^{*}$ be the unique equilibrium point of the system (2.1). Make a transformation $u(t)=x(t)-x^{*}$, then system (2.1) is transformed into

$$
\dot{u}(t)=\bar{\alpha}(u(t))\left[-\bar{\beta}(u(t))+W_{0} \bar{g}(u(t))+W_{1} \bar{g}(u(t-\tau))+W_{2} \int_{t-\tau}^{t} \bar{g}(u(s)) d s\right],
$$

where

$$
\begin{aligned}
& \bar{\alpha}(u(t))=\operatorname{diag}\left(\bar{\alpha}_{1}\left(u_{1}(t)\right), \bar{\alpha}_{2}\left(u_{2}(t)\right), \ldots, \bar{\alpha}_{n}\left(u_{n}(t)\right)\right), \\
& \bar{\beta}(u(t))=\left(\bar{\beta}_{1}\left(u_{1}(t)\right), \bar{\beta}_{2}\left(u_{2}(t)\right), \ldots, \bar{\beta}_{n}\left(u_{n}(t)\right)\right)^{T}, \\
& \bar{g}(u(t))=\left(\bar{g}_{1}\left(u_{1}(t)\right), \bar{g}_{2}\left(u_{2}(t)\right), \ldots, \bar{g}_{n}\left(u_{n}(t)\right)\right)^{T}, \\
& \bar{\alpha}_{i}\left(u_{i}(t)\right)=\alpha_{i}\left(u_{i}(t)+x_{i}^{*}\right), \\
& \bar{\beta}_{i}\left(u_{i}(t)\right)=\beta_{i}\left(u_{i}(t)+x_{i}^{*}\right)-\beta_{i}\left(x_{i}^{*}\right), \\
& \bar{g}_{i}\left(u_{i}(t)\right)=g_{i}\left(u_{i}(t)+x_{i}^{*}\right)-g_{i}\left(x_{i}^{*}\right), \quad i=1,2, \ldots, n .
\end{aligned}
$$

Similarly to (3.14), from Lemma 2.8, we have

$$
\bar{\beta}(u)=\beta\left(u+x^{*}\right)-\beta\left(x^{*}\right)=\bar{B} u,
$$

where $\bar{B} \in \cup_{z \in\left[x^{*}, u+x^{*}\right]} \partial \beta(z), \bar{B}=\operatorname{diag}\left(\bar{\beta}_{1}, \bar{\beta}_{2}, \ldots, \bar{\beta}_{n}\right)$ with $\bar{\beta}_{i} \geq \beta_{i}, i=1,2, \ldots, n$. 
Consider the following Lyapunov-Krasovskii functional candidate

$$
V(t)=V_{1}(t)+V_{2}(t)+V_{3}(t)+V_{4}(t)
$$

where

$$
\begin{aligned}
& V_{1}(t)=2 e^{\gamma t} \sum_{i=1}^{n} p_{i} \int_{0}^{u_{i}(t)} \frac{s}{\bar{\alpha}_{i}(s)} d s, \\
& V_{2}(t)=2 e^{\gamma t} \sum_{i=1}^{n} m_{i} \int_{0}^{u_{i}(t)} \frac{\bar{g}_{i}(s)}{\bar{\alpha}_{i}(s)} d s, \\
& V_{3}(t)=\int_{t-\tau}^{t} \bar{g}(u(\theta))^{T} S \bar{g}(u(\theta)) e^{\gamma \theta} d \theta, \\
& V_{4}(t)=\int_{-\tau}^{0} \int_{t+\theta}^{t} e^{\gamma s} \bar{g}(u(s))^{T} T \bar{g}(u(s)) d s d \theta .
\end{aligned}
$$

Calculating the time derivative of $V(t)$ along the trajectories of the system $(3.16)$ on $\left[0, t^{*}(\varphi)\right)$ with (3.17), by the assumption $\left(H_{1}\right)$ and Lemma 2.12 , we have

$$
\begin{aligned}
\dot{V}_{1}(t) \leq & \gamma e^{\gamma t} u(t)^{T} P \underline{A}^{-1} u(t)+2 e^{\gamma t} u(t)^{T} P \\
& \times\left[-\bar{B} u(t)+W_{0} \bar{g}(u(t))+W_{1} \bar{g}(u(t-\tau(t)))+W_{2} \int_{t-\tau(t)}^{t} \bar{g}(u(s)) d s\right] \\
\leq & e^{\gamma t} u(t)^{T}\left(\gamma P \underline{A}^{-1}-2 P B\right) u(t)+2 e^{\gamma t} u(t)^{T} P W_{0} \bar{g}(u(t)) \\
& +2 e^{\gamma t} u(t)^{T} P W_{1} \bar{g}(u(t-\tau))+2 e^{\gamma t} u(t)^{T} P W_{2} \int_{t-\tau}^{t} \bar{g}(u(s)) d s, \\
\dot{V}_{2}(t) \leq & 2 \gamma e^{\gamma t} u(t)^{T} M \underline{A}^{-1} \bar{g}(u(t))+2 e^{\gamma t} \bar{g}(u(t))^{T} M \\
& \times\left[-\bar{B} u(t)+W_{0} \bar{g}(u(t))+W_{1} \bar{g}(u(t-\tau(t)))+W_{2} \int_{t-\tau(t)}^{t} \bar{g}(u(s)) d s\right] \\
\leq & 2 e^{\gamma t} u(t)^{T}\left(\gamma M \underline{A}^{-1}-M B\right) \bar{g}(u(t))+2 e^{\gamma t} \bar{g}(u(t))^{T} M W_{0} \bar{g}(u(t)) \\
& +2 e^{\gamma t} \bar{g}(u(t))^{T} M W_{1} \bar{g}(u(t-\tau))+2 e^{\gamma t} \bar{g}(u(t))^{T} M W_{2} \int_{t-\tau}^{t} \bar{g}(u(s)) d s,
\end{aligned}
$$


Discrete Dynamics in Nature and Society

$$
\begin{aligned}
\dot{V}_{3}(t) & =e^{\gamma t} \bar{g}(u(t))^{T} S \bar{g}(u(t))-e^{\gamma t} e^{-\gamma \tau} \bar{g}(u(t-\tau))^{T} S \bar{g}(u(t-\tau)) \\
\dot{V}_{4}(t) & \leq \tau e^{\gamma t} \bar{g}(u(t))^{T} T \bar{g}(u(t))-\int_{t-\tau}^{t} e^{r s} \bar{g}(u(s))^{T} T \bar{g}(u(s)) d s \\
& \leq \tau e^{\gamma t} \bar{g}(u(t))^{T} T \bar{g}(u(t))-e^{\gamma(t-\tau)} \int_{t-\tau}^{t} \bar{g}(u(s))^{T} T \bar{g}(u(s)) d s \\
& \leq \tau e^{\gamma t} \bar{g}(u(t))^{T} T \bar{g}(u(t))-e^{\gamma(t-\tau)} \frac{1}{\tau}\left(\int_{t-\tau}^{t} \bar{g}(u(s))^{T} d s\right) T\left(\int_{t-\tau}^{t} \bar{g}(u(s)) d s\right) .
\end{aligned}
$$

Let $\xi(t)=\left[u(t)^{T}, \bar{g}(u(t))^{T}, \bar{g}(u(t-\tau))^{T},\left(\int_{t-\tau}^{t} \bar{g}(u(s)) d s\right)^{T}\right]^{T}$. By (3.17) and (3.20), we can obtain

$$
\dot{V}(t) \leq e^{\gamma \tau} \xi^{T}(t)\left[\begin{array}{cccc}
\gamma P \underline{A}^{-1}-P B-B P & P W_{0}+\gamma M \underline{A}^{-1}-M B & P W_{1} & P W_{2} \\
W_{0}^{T} \underline{P}+\gamma \underline{A}^{-1} M-B M & M W_{0}+W_{0}^{T} \bar{M}+S+\tau T & M W_{1} & M W_{2} \\
W_{1}^{T} P & W_{1}^{T} M & -\mathrm{e}^{-\gamma \tau} S & 0 \\
W_{2}^{T} P & W_{2}^{T} M & 0 & -\frac{\mathrm{e}^{-\gamma \tau}}{\tau} T
\end{array}\right] \xi(t)
$$

This implies $V(t) \leq V(0), t \in\left[0, t^{*}(\varphi)\right)$. Furthermore, from (3.18), it follows that

$$
2 \sum_{i=1}^{n} \frac{m_{i}}{\bar{\alpha}_{i}} \int_{0}^{u_{i}(t)} \bar{g}_{i}(s) d s \leq 2 \sum_{i=1}^{n} m_{i} \int_{0}^{u_{i}(t)} \frac{\bar{g}_{i}(s)}{\bar{\alpha}_{i}(s)} d s \leq V(0) e^{-\gamma t} \leq V(0)
$$

By (3.22) and Lemma 2.6, it is easy to derive that $u_{i}(t), i=1,2, \ldots, n$, are bounded on $\left[0, t^{*}\right)$. By virtue of the continuous theorem [47], $t^{*}(\varphi)=+\infty$. such that

Since $\bar{g}_{i}(s) \in \mathcal{J \ell}(\alpha), \bar{g}_{i}(0)=0$, by Lemma 2.7, there exist constants $\bar{q}_{i_{0}}>0$ and $\bar{r}_{i_{0}}>0$,

$$
\left|\bar{g}_{i}(s)\right| \geq \bar{q}_{i_{0}}|s|^{\alpha}, \quad \forall|s| \leq \bar{r}_{i_{0}}, \quad i=1,2, \ldots, n
$$

Moreover, from (3.22), we can get that

$$
\lim _{t \rightarrow+\infty} u_{i}(t)=0, \quad i=1,2, \ldots, n .
$$


Thus there exists a scalar $\tau>0$, when $t \geq \tau, \quad u_{i}(t) \in\left[-\bar{r}_{0}, \bar{r}_{0}\right]$, where $\bar{r}_{0}=\min _{1 \leq i \leq n} \bar{r}_{i_{0}}$. Let $m=\min _{1 \leq i \leq n} m_{i}, \bar{\alpha}=\max _{1 \leq i \leq n} \bar{\alpha}_{i}$, and $\bar{q}_{0}=\min _{1 \leq i \leq n} \bar{q}_{i_{0}}$. From (3.22) and (3.23), we have

$$
\begin{aligned}
\frac{V(0)}{2} e^{-\gamma t} & \geq \sum_{i=1}^{n} \frac{m_{i}}{\bar{\alpha}_{i}} \int_{0}^{u_{i}(t)} \bar{g}_{i}(s) d s \\
& \geq \sum_{i=1}^{n} \frac{m_{i}}{\bar{\alpha}_{i}} \int_{0}^{\left|u_{i}(t)\right|} \bar{q}_{i_{0}}|s|^{\alpha} d s \\
& \geq \sum_{i=1}^{n} \frac{m_{i}}{\bar{\alpha}_{i}} \int_{0}^{\left|u_{i}(t)\right|} \bar{q}_{0}|s|^{\alpha} d s \\
& \geq \frac{m \bar{q}_{0}}{\bar{\alpha}(\alpha+1)}\left\{\max _{1 \leq i \leq n}\left|u_{i}(t)\right|\right\}^{\alpha+1}, \quad t \geq \tau .
\end{aligned}
$$

That is, when $t \geq \tau$,

$$
\max _{1 \leq i \leq n}\left|u_{i}(t)\right| \leq\left[\frac{\bar{\alpha}(\alpha+1) V(0)}{2 m \bar{q}_{0}}\right]^{1 /(1+\alpha)} e^{-(\gamma /(1+\alpha)) t}
$$

where

$$
\begin{aligned}
V(0)= & 2 \sum_{i=1}^{n} p_{i} \int_{0}^{\varphi_{i}(0)-x_{i}^{*}} \frac{s}{\overline{\alpha_{i}(s)}} d s+2 \sum_{i=1}^{n} m_{i} \int_{0}^{\varphi_{i}(0)-x_{i}^{*}} \frac{\bar{g}_{i}(s)}{\overline{\alpha_{i}(s)}} d s \\
& +\int_{-\tau}^{0} \bar{g}\left(\varphi(\theta)-x^{*}\right)^{T} S \bar{g}\left(\varphi(\theta)-x^{*}\right) e^{\gamma \theta} d \theta \\
& +\int_{-\tau}^{0} \int_{\theta}^{0} e^{\gamma s} \bar{g}\left(\varphi(s)-x^{*}\right)^{T} T \bar{g}\left(\varphi(s)-x^{*}\right) d s d \theta .
\end{aligned}
$$

Let $\eta=\sqrt{n}\left[\bar{\alpha}(\alpha+1) V(0) / 2 m \bar{q}_{0}\right]^{1 /(1+\alpha)}$. By (3.26), $\left\|x(t)-x^{*}\right\| \leq \eta e^{-(\gamma /(1+\alpha)) t}$, for all $t \geq \tau$. This shows that the equilibrium point of the system (2.1) is globally exponentially stable. This completes the proof of Theorem 3.1.

\section{Stability of the Switched Cohen-Grossberg Neural Network}

The switched Cohen-Grossberg neural networks with mixed time delays consist of a set of Cohen-Grossberg neural networks with mixed time delays and a switching rule. Each of the Cohen-Grossberg neural networks with mixed time delays is regarded as an individual subsystem. The operation mode of the switched neural networks is determined by the switching rule. In the following, we will develop the switched Cohen-Grossberg neural networks model with mixed time delays. 


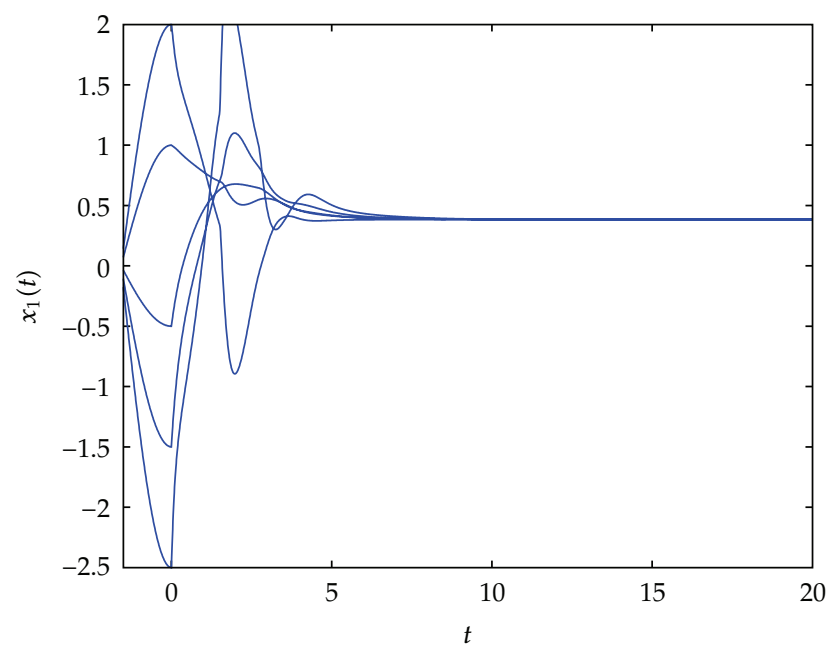

Figure 1: The convergence of the state $x_{1}(t)$ of the network in Example 5.1.

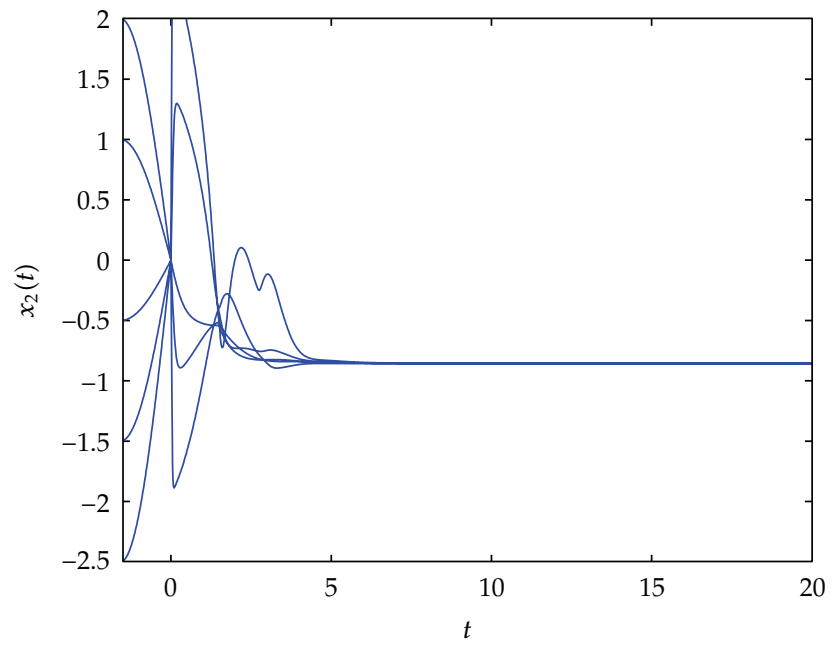

Figure 2: The convergence of the state $x_{2}(t)$ of the network in Example 5.1.

Suppose that $x^{*}$ is the unique equilibrium point of the system (2.1). Similar to (3.16), make a transformation $u(t)=x(t)-x^{*}$, then system (2.1) is transformed into

$$
\dot{u}(t)=\bar{\alpha}(u(t))\left[-\bar{\beta}(u(t))+W_{0} \bar{g}(u(t))+W_{1} \bar{g}(u(t-\tau))+W_{2} \int_{t-\tau}^{t} \bar{g}(u(s)) d s\right] .
$$




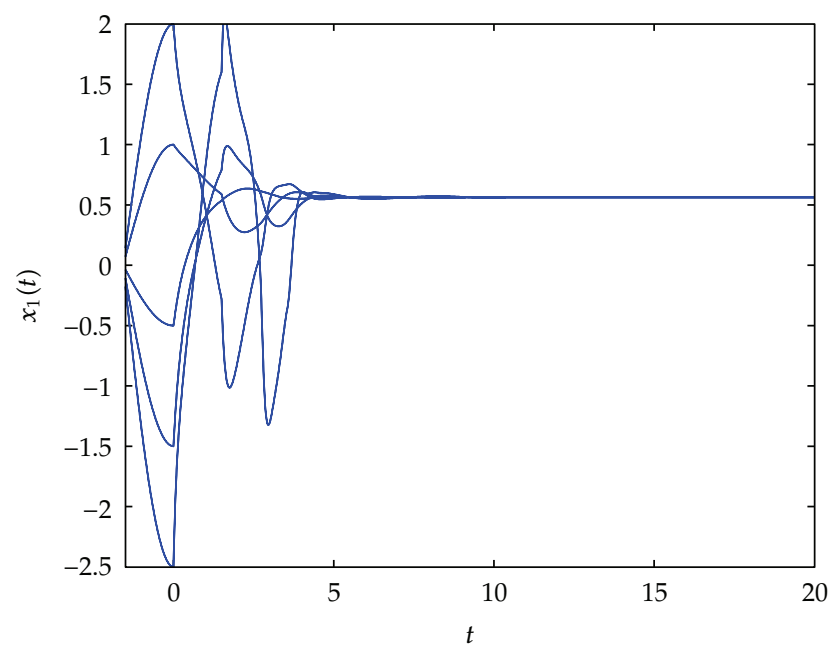

Figure 3: The convergence of the state $x_{1}(t)$ of the network in Example 5.2.

The switched Cohen-Grossberg neural networks with mixed time delays can be described as

$$
\dot{u}(t)=\bar{\alpha}(u(t))\left[-\bar{\beta}(u(t))+W_{0_{\sigma}(t)} \bar{g}(u(t))+W_{1_{\sigma}(t)} \bar{g}(u(t-\tau))+W_{2_{\sigma}(t)} \int_{t-\tau}^{t} \bar{g}(u(s)) d s\right]
$$

where $\sigma(t):[0,+\infty) \rightarrow \Gamma=\{1,2, \ldots, N\}$ is the switching signal, which is a piecewise constant function of time. This means that the matrices $\left(W_{\sigma_{\sigma(t)}}, W_{1_{\sigma(t)}}, W_{2_{\sigma(t)}}, J_{\sigma(t)}\right)$ are allowed to take values, at an arbitrary time, in the finite set $\left\{\left(W_{0_{1}}, W_{1_{1}}, W_{2_{1}}, J_{1}\right),\left(W_{0_{2}}, W_{1_{2}}, W_{2_{2}}, J_{2}\right), \ldots,\left(W_{0_{N}}, W_{1_{N}}, W_{2_{N}}, J_{N}\right)\right\}$. The initial value associated with (4.3) is assumed to be $u(s)=\tilde{\varphi}(s), \tilde{\varphi}(s)$ is a continuous function on $[-\tau, 0]$.

In this paper, it is assumed that the switching rule $\sigma$ is not known a priori and its instantaneous value is available in real time. Corresponding to the switching signal $\sigma(t)$, we have the switching sequence $\left\{x_{t_{0}} ;\left(i_{0}, t_{0}\right), \ldots,\left(i_{k}, t_{k}\right), \ldots, \mid i_{k} \in \Gamma, k=0,1, \ldots\right\}$, which means that the $i_{k}$ th subsystem is activated when $t \in\left[t_{k}, t_{k+1}\right)$.

In the following, we will consider the switched Cohen-Grossberg neural networks with mixed time delays in (4.2). The average dwell time approach will be used to derive the exponential stability of the network.

Theorem 4.1. Under the assumptions $\left(H_{1}\right)--\left(H_{3}\right)$, if there exist positive definite matrices $S_{i}, T_{i}$, positive definite diagonal matrices $M_{i}, P_{i}$ and scalars $\gamma>0, \mu \geq 1$ such that

$$
\left[\begin{array}{cccc}
\gamma P \underline{A}^{-1}-P_{i} B_{i}-B_{i} P_{i} & P_{i} W_{0_{i}}+\gamma M_{i} \underline{A}^{-1}-M_{i} B_{i} & P_{i} W_{1_{i}} & P_{i} W_{2_{i}} \\
W_{0_{i}}^{T} P_{i}+\gamma \underline{A}^{-1} M_{i}-B_{i} M_{i} & M_{i} W_{0_{i}}+W_{0_{i}}^{T} M_{i}+S_{i}+\tau T_{i} & M_{i} W_{1_{i}} & M_{i} W_{2_{i}} \\
W_{1_{i}}^{T} P_{i} & W_{1_{i}}^{T} M_{i} & -e^{-\gamma \tau} S_{i} & 0 \\
W_{2_{i}}^{T} P_{i} & W_{2_{i}}^{T} M_{i} & 0 & -\frac{e^{-\gamma \tau}}{\tau} T_{i}
\end{array}\right]<0,
$$


Discrete Dynamics in Nature and Society

$$
P_{i} \leq \mu P_{j}, \quad M_{i} \leq \mu M_{j}, \quad S_{i} \leq \mu S_{j}, \quad T_{i} \leq \mu T_{j}, \quad \forall i, j \in \Gamma,
$$

where $M_{i}=\operatorname{diag}\left(m_{i 1}, m_{i 2}, \ldots, m_{i n}\right), P_{i}=\operatorname{diag}\left(p_{i 1}, p_{i 2}, \ldots, p_{i n}\right)$, then the switched Cohen-Grossberg neural network (4.2) is globally exponentially stable for any switching signal with average dwell time satisfying

$$
T_{a}>T_{a}^{*}=(1+\alpha) \frac{\ln \mu}{\gamma}
$$

Moreover, an estimate of the state decay for the system (4.2) is given by

$$
\|u(t)\| \leq \sqrt{n}\left\{e^{-\gamma t_{0}} \frac{\bar{\alpha}(\alpha+1)}{2 m \bar{q}_{0}}\right\}^{1 /(1+\alpha)} \max _{i \in \Gamma} V_{i}\left(t_{0}\right) e^{-\left(\gamma /(1+\alpha)-(\ln \mu) / T_{a}\right)\left(t-t_{0}\right)}, \quad t>t_{0} .
$$

Proof. Consider the multiple Lyapunov-Krasovskii functional candidate

$$
V_{\sigma(t)}(t)=V_{1_{\sigma(t)}}(t)+V_{2_{\sigma(t)}}(t)+V_{3_{\sigma(t)}}(t)+V_{4_{\sigma(t)}}(t),
$$

where

$$
\begin{aligned}
& V_{1_{\sigma(t)}}(t)=2 e^{\gamma t} \sum_{j=1}^{n} p_{\sigma(t) j} \int_{0}^{u_{j}(t)} \frac{s}{\bar{\alpha}_{j}(s)} d s, \\
& V_{2_{\sigma(t)}}(t)=2 e^{\gamma t} \sum_{j=1}^{n} m_{\sigma(t) j} \int_{0}^{u_{j}(t)} \frac{\bar{g}_{j}(s)}{\bar{\alpha}_{j}(s)} d s, \\
& V_{3_{\sigma(t)}}(t)=\int_{t-\tau}^{t} \bar{g}(u(\theta))^{T} S_{\sigma(t)} \bar{g}(u(\theta)) e^{\gamma \theta} d \theta, \\
& V_{4_{\sigma(t)}}(t)=\int_{-\tau}^{0} \int_{t+\theta}^{t} e^{\gamma s} \bar{g}(u(s))^{T} T_{\sigma(t)} \bar{g}(u(s)) d s d \theta .
\end{aligned}
$$

When $t \in\left[t_{k}, t_{k+1}\right)$, the $i_{k}$ th subsystem is activated. Arguing as in the proof Theorem 3.1, we can get $\dot{V}_{\sigma(t)}(t) \leq 0$. Thus, $V_{\sigma(t)}(t) \leq V_{\sigma\left(t_{k}\right)}\left(t_{k}\right)$. In the light of (4.4) and (4.7), it follows that $V_{\sigma\left(t_{k}\right)}\left(t_{k}\right) \leq \mu V_{\sigma\left(t_{k}^{-}\right)}\left(t_{k}^{-}\right)$. Therefore, when $t \in\left[t_{k}, t_{k+1}\right)$, we have

$$
\begin{aligned}
V_{\sigma(t)}(t) & \leq V_{\sigma\left(t_{k}\right)}\left(t_{k}\right) \leq \mu V_{\sigma\left(t_{k}^{-}\right)}\left(t_{k}^{-}\right) \\
& \leq \mu V_{\sigma\left(t_{k-1}\right)}\left(t_{k-1}\right) \leq \mu^{2} V_{\sigma\left(t_{k-1}^{-}\right)}\left(t_{k-1}^{-}\right) \\
& \leq \mu^{2} V_{\sigma\left(t_{k-2}\right)}\left(t_{k-2}\right) \leq \cdots \leq \mu^{k} V_{\sigma\left(t_{0}\right)}\left(t_{0}\right) .
\end{aligned}
$$


By (4.9); there exist constants $\tau>t_{0}$, when $t \geq \tau$, we have

$$
\begin{aligned}
\frac{V_{\sigma\left(t_{0}\right)}\left(t_{0}\right)}{2} \mu^{k} e^{-\gamma t} & \geq \sum_{j=1}^{n} \frac{m_{\sigma(t) j}}{\bar{\alpha}_{j}} \int_{0}^{u_{j}(t)} \bar{g}_{i}(s) d s \\
& \geq \sum_{j=1}^{n} \frac{m_{\sigma(t) j}}{\bar{\alpha}_{j}} \int_{0}^{\left|u_{i}(t)\right|} \bar{q}_{i_{0}}|s|^{\alpha} d s \\
& \geq \sum_{j=1}^{n} \frac{m_{\sigma(t) j}}{\bar{\alpha}_{j}} \int_{0}^{\left|u_{i}(t)\right|} \bar{q}_{0}|s|^{\alpha} d s \\
& \geq \frac{m \bar{q}_{0}}{\bar{\alpha}(\alpha+1)}\left\{\max _{1 \leq i \leq n}\left|u_{i}(t)\right|\right\}^{\alpha+1},
\end{aligned}
$$

where $m=\min _{j \in \Gamma, 1 \leq i \leq n} m_{j i}, \bar{\alpha}=\max _{1 \leq i \leq n} \bar{\alpha}_{i}$ and $\bar{q}_{0}=\min _{1 \leq i \leq n} \bar{q}_{i_{0}}$. Hence,

$$
\max _{1 \leq i \leq n}\left|u_{i}(t)\right| \leq\left\{\frac{\bar{\alpha}(\alpha+1) V_{\sigma\left(t_{0}\right)}\left(t_{0}\right)}{2 m \bar{q}_{0}}\right\}^{1 /(1+\alpha)} \mu^{k} e^{-(\gamma /(1+\alpha)) t}
$$

This implies that

$$
\|u(t)\| \leq \sqrt{n}\left\{\frac{\bar{\alpha}(\alpha+1) V_{\sigma\left(t_{0}\right)}\left(t_{0}\right)}{2 m \bar{q}_{0}}\right\}^{1 /(1+\alpha)} \mu^{k} e^{-(\gamma /(1+\alpha)) t}
$$

Due to $k \leq\left(t-t_{0}\right) / T_{a}$ and $T_{a}>T_{a}^{*}=(1+\alpha)((\ln \mu) / \gamma)$, then we can get

$$
\begin{aligned}
\|u(t)\| & \leq \sqrt{n}\left\{e^{-\gamma t_{0}} \frac{\bar{\alpha}(\alpha+1)}{2 m \bar{q}_{0}}\right\}^{1 /(1+\alpha)} \max _{i \in \Gamma} V_{i}\left(t_{0}\right) \mu^{k} e^{-(\gamma /(1+\alpha))\left(t-t_{0}\right)} \\
& =\sqrt{n}\left\{e^{-\gamma t_{0}} \frac{\bar{\alpha}(\alpha+1)}{2 m \bar{q}_{0}}\right\}^{1 /(1+\alpha)} \max _{i \in \Gamma} V_{i}\left(t_{0}\right) e^{k \ln \mu} e^{-(\gamma /(1+\alpha))\left(t-t_{0}\right)} \\
& \leq \sqrt{n}\left\{e^{-\gamma t_{0}} \frac{\bar{\alpha}(\alpha+1)}{2 m \bar{q}_{0}}\right\}^{1 /(1+\alpha)} \max _{i \in \Gamma} V_{i}\left(t_{0}\right) e^{-\left(\gamma /(1+\alpha)-(\ln \mu) / T_{a}\right)\left(t-t_{0}\right)}, \quad t>t_{0} .
\end{aligned}
$$

This implies that the switched Cohen-Grossberg neural network (4.2) is globally exponentially stable. The proof is completed.

Remark 4.2. It is clear that, according to Theorem 4.1, the delay-dependent stability of the considered neural networks is dependent on $\mu$ for given $\gamma$. If $\mu=1$, we have from (4.5) that the switching signal can be arbitrary, and (4.4) reduces to $P_{i} \leq P_{j}, M_{i} \leq M_{j}, S_{i} \leq S_{j}, T_{i} \leq$ $T_{j}$, for all $i, j \in \Gamma$, which implies $P_{i}=P_{j}, M_{i}=M_{j}, S_{i}=S_{j}, T_{i}=T_{j}$, for all $i, j \in \Gamma$, which means that it requires a common Lyapunov functional for all subsystems. If $\mu \rightarrow+\infty$, we get from (4.5) that there is no switching, that is, switching signal will have a great dwelltime on the average. 
Remark 4.3. In the existing literature $[2,3,17,22,27-29,34-38]$, the activation functions of switched neural networks are required to be Lipschitz continuous. However, in this paper, the activation function is inverse Hölder function. It is obvious the results of this paper are different from the results in the above literatures. Hence, the work in this paper is an extension of the scope of the current investigation in this field.

\section{Illustrative Examples}

In this section, two illustrative examples will be given to check the validity of the results obtained in Theorems 3.1 and 4.1 .

Example 5.1. Consider a second-order Cohen-Grossberg neural networks with mixed time delays in (2.1) with the following parameters:

$$
W_{0}=\left(\begin{array}{cc}
-9999 & 0.009 \\
0.0001 & -9999
\end{array}\right), \quad W_{1}=\left(\begin{array}{cc}
0.25 & 0.03 \\
0.01 & 0.25
\end{array}\right), \quad W_{2}=\left(\begin{array}{cc}
0.05 & 0.03 \\
0.02 & 0.45
\end{array}\right), \quad J=(0,0)^{T} .
$$

Set $\alpha_{i}(\theta)=2+\sin \theta, \quad \beta_{i}(\theta)=\left\{\begin{array}{cc}\theta, & \theta \geq 0, \\ 2 \theta, & \theta \leq 0,\end{array}\right.$. The activation functions are taken as $g_{i}(\theta)=\theta^{3}$, $i=1,2$, and $\tau=1$.

It is easy to check that assumptions $\left(H_{1}\right)--\left(H_{3}\right)$ hold. $\beta_{i}(\theta)$ is locally Lipschitz; $\underline{A}$ and $B$ is the second-order identity matrix.

Take $\gamma=0.3$. Solving the LMI in (3.1) by using appropriate LMI solver in the Matlab, the feasible positive definite matrices $P, M, S, T$ could be as

$$
\begin{aligned}
& P=\left(\begin{array}{cc}
0.0017 & 0 \\
0 & 0.0017
\end{array}\right), \quad M=\left(\begin{array}{cc}
23.3316 & 0 \\
0 & 23.3316
\end{array}\right) \\
& S=\left(\begin{array}{cc}
3.4137 & -0.0019 \\
-0.0019 & 3.4137
\end{array}\right), \quad T=\left(\begin{array}{cc}
3.4137 & -0.0019 \\
-0.0019 & 3.4137
\end{array}\right) .
\end{aligned}
$$

All assumptions of Theorem 3.1 hold. Hence, this neural network has one unique equilibrium point, which is globally exponentially stable.

Figures 1 and 2 display the state trajectories of this neural network with initial values $\varphi(t)=(\cos t, \sin t)^{T},(0.5 \cos t,-0.5 \sin t)^{T},(-1.5 \cos t, 1.5 \sin t)^{T},(2 \cos t, 2 \sin t)^{T}$ and $(-2.5 \cos t, 2.5 \sin t)^{T}, t \in[-1,0]$. It can be seen that these trajectories converge to the unique equilibrium $x^{*}=(0,0)^{T}$ of the network. This is in accordance with the conclusion of Theorem 3.1. 


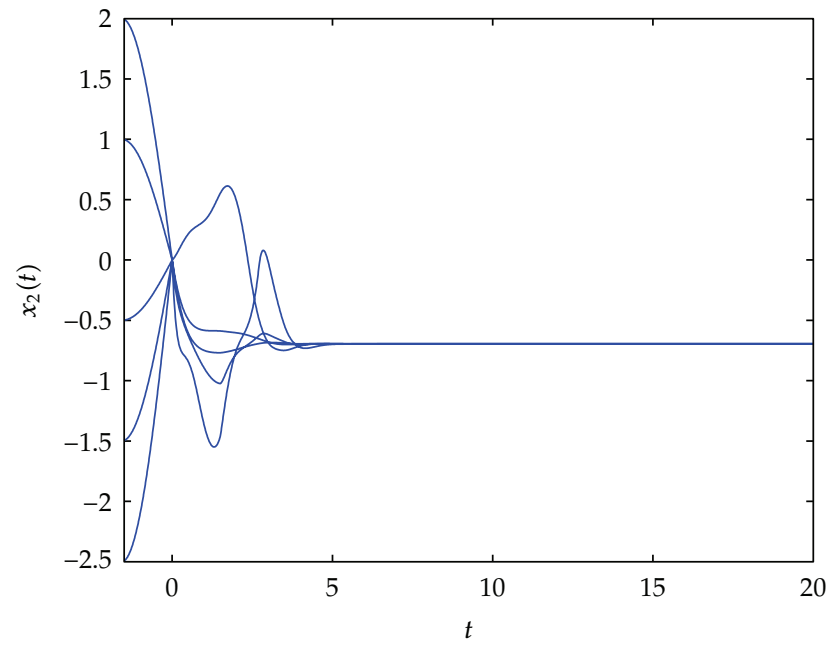

Figure 4: The convergence of the state $x_{2}(t)$ of the network in Example 5.2.

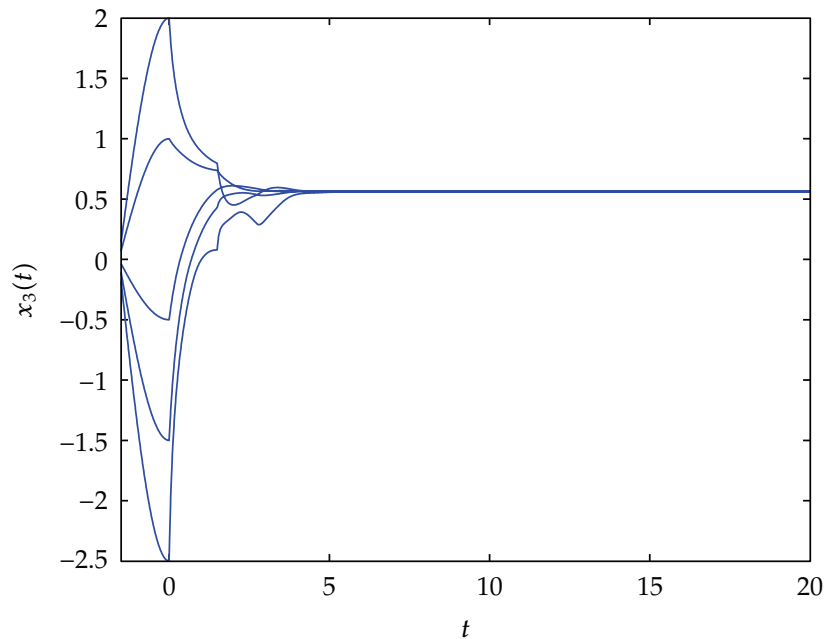

Figure 5: The convergence of the state $x_{3}(t)$ of the network in Example 5.2. 
Example 5.2. Consider a third-order switched Cohen-Grossberg neural networks with mixed time delays in (4.3) with the switching signal $\sigma(\mathrm{t}):[0,+\infty) \rightarrow \Gamma=\{1,2\}$ and the following parameters:

$$
\begin{aligned}
& W_{0_{1}}=\left(\begin{array}{ccc}
-832.651 & 3.13 & 0.51 \\
6.01 & -807.439 & 3.52 \\
2.03 & 3.05 & -478.954
\end{array}\right), \quad W_{0_{2}}=\left(\begin{array}{ccc}
-4959 & 4.03 & 3.31 \\
0.01 & -8079 & 2.82 \\
8.03 & 6.05 & -9278
\end{array}\right), \\
& W_{1_{1}}=\left(\begin{array}{ccc}
0.25 & 0.16 & 0.03 \\
0.01 & 0.32 & 0.25 \\
0.21 & 0.32 & 0.06
\end{array}\right), \quad W_{1_{2}}=\left(\begin{array}{lll}
0.25 & 0.16 & 0.03 \\
0.01 & 0.32 & 4.25 \\
0.21 & 0.32 & 0.06
\end{array}\right), \\
& W_{2_{1}}=\left(\begin{array}{ccc}
0.95 & 1.04 & 0.03 \\
1.02 & 1.03 & 1.45 \\
20.04 & 5.02 & 80.37
\end{array}\right),
\end{aligned}
$$

Set $\alpha_{i}(\theta)=2-\cos \theta, \beta_{i}(\theta)$ are the functions in Example 5.1, and the activation functions are taken as $g_{i}(\theta)=\theta^{3}+\theta, i=1,2,3$, and $\tau=1$.

It is easy to check that assumptions $\left(H_{1}\right)--\left(H_{3}\right)$ hold; $\underline{A}$ and $B$ is the third-order identity matrix.

Take $\gamma=0.1, \mu=1.2$. Solving the LMIs in (4.3) and (4.4) by using appropriate LMI solver in the Matlab, the feasible positive definite matrices $P_{1}, P_{2}, M_{1}, M_{2}, S_{1}, S_{2}, T_{1}, T_{2}$ could be as

$$
\begin{aligned}
& P_{1}=\left(\begin{array}{ccc}
0.6334 & 0 & 0 \\
0 & 0.6334 & 0 \\
0 & 0 & 0.6334
\end{array}\right), \quad M_{1}=\left(\begin{array}{ccc}
0.0418 & 0 & 0 \\
0 & 0.0418 & 0 \\
0 & 0 & 0.0418
\end{array}\right), \\
& S_{1}=\left(\begin{array}{ccc}
0.7500 & 0.0182 & -0.0685 \\
0.0182 & 0.8893 & -0.0165 \\
-0.0685 & -0.0165 & 0.2732
\end{array}\right), \quad T_{1}=\left(\begin{array}{ccc}
0.7811 & 0.0266 & 0.0679 \\
0.0266 & 0.8895 & 0.0183 \\
0.0679 & 0.0183 & 0.8362
\end{array}\right), \\
& P_{2}=\left(\begin{array}{ccc}
0.6620 & 0 & 0 \\
0 & 0.6620 & 0 \\
0 & 0 & 0.6620
\end{array}\right), \quad M_{2}=\left(\begin{array}{ccc}
0.0365 & 0 & 0 \\
0 & 0.0365 & 0 \\
0 & 0 & 0.0365
\end{array}\right) \text {, } \\
& S_{2}=\left(\begin{array}{ccc}
0.7855 & 0.0191 & -0.0717 \\
0.0191 & 0.9312 & -0.0173 \\
-0.0717 & -0.0173 & 0.2862
\end{array}\right), \quad T_{2}=\left(\begin{array}{ccc}
0.4710 & 0.0161 & 0.0411 \\
0.0161 & 0.5368 & 0.0111 \\
0.0411 & 0.0111 & 0.5045
\end{array}\right)
\end{aligned}
$$

By using (4.5), it follows that the average dwell time $T_{a}^{*}=3.6461$. All the assumptions of Theorem 4.1 hold. Hence, this switched neural network is globally exponentially stable.

For numerical simulation, assume that the two subsystems are switched every four seconds. Figures 3,4 , and 5 display the state trajectories of this neural network with initial values $\widetilde{\varphi}(t)=$ $(-0.5 \cos t, 0.5 \sin t, 0.5 \cos t)^{T},(\cos t,-\sin t,-\cos t)^{T},(-1.5 \cos t, 1.5 \sin t, 1.5 \cos t)^{T}$, 
$(-2 \cos t, 2 \sin t, 2 \sin t)^{T}$ and $(2.5 \cos t,-2.5 \sin t,-2.5 \cos t)^{T}, t \in[-1,0]$. It can be seen that these trajectories converge to the unique equilibrium $u^{*}=(0,0,0)^{T}$ of the network. This is in accordance with the conclusion of Theorem 4.1.

\section{Conclusion}

In this paper, the existence, uniqueness, and global stability of the equilibrium point for Cohen-Grossberg neural networks with mixed time delays, $\alpha$-inverse Hölder neuron activation functions, and nonsmooth behaved functions have been discussed. By applying multiple Lyapunov-Krasovskii functional, a delay-dependent global exponential stability criterion has been obtained in terms of LMIs for the switched Cohen-Grossberg neural networks with mixed time delays and $\alpha$-inverse Hölder neuron activation functions under the switching rule with the average dwell time property. The results obtained are easily checked and applied in practice engineering.

When neuron activation functions are non-Lipschitz functions, it is possible that the neural network system has not the global solution and the equilibrium point. This leads to difficulty in solving the stability problem, particularly exponential stability for the switched neural networks with non-Lipschitz activation functions. In the future, the stability problem for the switched neural networks with other non-Lipschitz activation functions will be expected to be solved.

\section{Acknowledgments}

This work was supported by the Natural Science Foundation of Hebei Province of China (A2011203103) and the Hebei Province Education Foundation of China (2009157).

\section{References}

[1] H. Wu, L. He, L. Qin, T. Feng, and R. Shi, "Solving interval quadratic program with box set constraints in engineering by a projection neural network," Information Technology Journal, vol. 9, no. 8, pp. 16151621, 2010.

[2] C. Y. Lu, W. J. Shyr, K. C. Yao, C. W. Liao, and C. K. Huang, “Delay-dependent Hoo control for discretetime uncertain recurrent neural networks with intrerval time-varying delay," International Journal of Innovative Computing, Information and Control, vol. 5, no. 10, pp. 3483-3493, 2009.

[3] X. Luan, P. Shi, and F. Liu, "Robust adaptive control for greenhouse climate using neural networks," International Journal of Robust and Nonlinear Control, vol. 21, no. 7, pp. 815-826, 2011.

[4] R. Mei, Q. Wu, and C. Jiang, "Neural network robust adaptive control for a class of time delay uncertain nonlinear systems," International Journal of Innovative Computing, Information and Control, vol. 3, pp. 931-940, 2010.

[5] X. Dong, Y. Zhao, Y. Xu, Z. Zhang, and P. Shi, "Design of PSO fuzzy neural network control for ball and plate system," International Journal of Innovative Computing, Information and Control, vol. 7, pp. 7091-7103, 2011.

[6] M. A. Cohen and S. Grossberg, "Absolute stability of global pattern formation and parallel memory storage by competitive neural networks," IEEE Transactions on Systems, Man, and Cybernetics, vol. 13, no. 5, pp. 815-826, 1983.

[7] S. Grossberg, "Nonlinear neural networks principles, mechanisms, and architectures," Neural Networks, vol. 1, pp. 17-66, 1988.

[8] J. Cao and X. Li, "Stability in delayed Cohen-Grossberg neural networks: LMI optimization approach," Physica D, vol. 212, no. 1-2, pp. 54-65, 2005.

[9] Y. Chen, "Global asymptotic stability of delayed Cohen-Grossberg neural networks," IEEE 
Transactions on Circuits and Systems I, vol. 53, no. 2, pp. 351-357, 2006.

[10] Z. Wang, Y. Liu, M. Li, and X. Liu, "Stability analysis for stochastic Cohen-Grossberg neural networks with mixed time delays," IEEE Transactions on Neural Networks, vol. 17, pp. 814-820, 2006.

[11] C. Huang and L. Huang, "Dynamics of a class of Cohen-Grossberg neural networks with timevarying delays," Nonlinear Analysis. Real World Applications, vol. 8, no. 1, pp. 40-52, 2007.

[12] X. Yang, C. Huang, D. Zhang, and Y. Long, "Dynamics of Cohen-Grossberg neural networks with mixed delays and impulses," Abstract and Applied Analysis, vol. 2008, Article ID 432341, 14 pages, 2008.

[13] L. Hu, H. Gao, and P. Shi, "New stability criteria for Cohen-Grossberg neural networks with time delays," IET Control Theory \& Applications, vol. 3, no. 9, pp. 1275-1282, 2009.

[14] Z. Wang, H. Zhang, and W. Yu, "Robust stability of Cohen-Grossberg neural networks via state transmission matrix," IEEE Transactions on Neural Networks, vol. 20, pp. 169-174, 2009.

[15] Z. Wang, H. Zhang, and W. Yu, "Robust stability criteria for interval Cohen-Grossberg neural networks with time varying delay," Neurocomputing, vol. 72, pp. 1105-1110, 2009.

[16] H. Zhang, Z. Wang, and D. Liu, "Robust stability analysis for interval Cohen-Grossberg neural networks with unknown time-varying delays," IEEE Transactions on Neural Networks, vol. 19, pp. 1942-1955, 2008.

[17] J. Cao, K. Yuan, and H. Li, "Global asymptotical stability of recurrent neural networks with multiple discrete delays and distributed delays," IEEE Transactions on Neural Networks, vol. 17, pp. 1646-1651, 2006.

[18] H. Zhang, Z. Wang, and D. Liu, "Global asymptotic stability and robust stability of a class of CohenGrossberg neural networks with mixed delays," IEEE Transactions on Circuits and Systems I, vol. 56, no. 3, pp. 616-629, 2009.

[19] Q. Zhang, X. Wei, and J. Xu, "A generalized LMI-based approach to the global asymptotic stability of discrete-time delayed recurrent neural networks," International Journal of Innovative Computing, Information and Control, vol. 4, no. 6, pp. 1393-1400, 2008.

[20] R. S. Gau, C. H. Lien, and J. G. Hsieh, "Novel stability conditions for interval delayed neural networks with multiple time-varying delays," International Journal of Innovative Computing, Information and Control, vol. 7, no. 1, pp. 433-444, 2011.

[21] Z. Wu, P. Shi, H. Su, and J. Chu, "Passivity analysis for discrete-time stochastic Markovian jump neural networks with mixed time-delays," IEEE Transactions on Neural Networks, vol. 22, pp. $1566-$ 1575, 2011.

[22] R. Yang, Z. Zhang, and P. Shi, "Exponential stability on stochastic neural networks with discrete interval and distributed delays," IEEE Transactions on Neural Networks, vol. 21, no. 1, pp. 169-175, 2010.

[23] H. Wu and X. Xue, "Stability analysis for neural networks with inverse Lipschitzian neuron activations and impulses," Applied Mathematical Modelling, vol. 32, no. 11, pp. 2347-2359, 2008.

[24] H. Wu, "Global exponential stability of Hopfield neural networks with delays and inverse Lipschitz neuron activations," Nonlinear Analysis. Real World Applications, vol. 10, no. 4, pp. 2297-2306, 2009.

[25] X. Nie and J. Cao, "Stability analysis for the generalized Cohen-Grossberg neural networks with inverse Lipschitz neuron activations," Computers \& Mathematics with Applications, vol. 57, no. 9, pp. 1522-1536, 2009.

[26] J. Jing, Y. Li, and H. Wu, "Global exponential stability in cohen-grossberg neural networks withinverse hölder neuron activations and impulses on time scales," ICIC Express Letters, vol. 4, no. 3, pp. 647-652, 2010.

[27] D. Wang, W. Wang, and P. Shi, “Exponential $H_{\infty}$ filtering for switched linear systems with interval time-varying delay," International Journal of Robust and Nonlinear Control, vol. 19, no. 5, pp. 532-551, 2009.

[28] Y. Tsividis, "Switched neural networks," US Patent no. 4873661, 1989.

[29] T. X. Brown, "Neural networks for switching," IEEE Communications Magazine, vol. 27, pp. 72-81, 1989.

[30] M. Muselli, "Gene selection through switched neural networks," in Proceedings of the Network Tools and Applications in Biology Workshop, pp. 27-28, 2003.

[31] H. Huang, Y. Qu, and H. X. Li, "Robust stability analysis of switched Hopfield neural networks with time-varying delay under uncertainty," Physics Letters, Section A, vol. 345, no. 4-6, pp. 345-354, 2005.

[32] K. Yuan, J. Cao, and H. X. Li, "Robust stability of switched Cohen-Grossberg neural networks with mixed time-varying delays," IEEE Transactions on Systems, Man, and Cybernetics, Part B, vol. 36, no. 6, pp. 1356-1363, 2006. 
[33] P. Li and J. Cao, "Global stability in switched recurrent neural networks with time-varying delay via nonlinear measure," Nonlinear Dynamics, vol. 49, no. 1-2, pp. 295-305, 2007.

[34] X. Lou and B. Cui, "Delay-dependent criteria for global robust periodicity of uncertain switched recurrent neural networks with time-varying delay," IEEE Transactions on Neural Networks, vol. 19, pp. 549-557, 2008.

[35] H. Wu, N. Li, K. Wang, G. Xu, and Q. Guo, "Global robust stability of switched interval neural networks with discrete and distributed time-varying delays of neural type," Mathematical Problems in Engineering, vol. 2012, Article ID 361871, 18 pages, 2012.

[36] J. Lian and K. Zhang, "Exponential stability for switched Cohen-Grossberg neural networks with average dwell time," Nonlinear Dynamics, vol. 63, no. 3, pp. 331-343, 2011.

[37] Z. Wu, P. Shi, H. Su, and J. Chu, "Delay-dependent exponential stability analysis for discrete-time switched neural networks with time-varying delay," Neurocomputing, vol. 74, pp. 1626-1631, 2011.

[38] Z. Wu, P. Shi, H. Su, and J. Chu, "Delay-dependent stability analysis for switched neural networks with time-varying delay," IEEE Transactions on Systems, Man, and Cybernetics, vol. 41, pp. 1522-1530, 2011.

[39] J. Lian, Z. Feng, and P. Shi, “Observer design for switched recurrent neural networks: an average dwell time approach," IEEE Transactions on Neural Networks, vol. 22, pp. 1547-1556, 2011.

[40] M. S. Mahmoud, "Switched discrete-time systems with time-varying delays: a generalized $\mathrm{H}_{2}$ approach," Computers \& Mathematics with Applications, vol. 57, no. 1, pp. 79-95, 2009.

[41] W. Zhang and L. Yu, "Stability analysis for discrete-time switched time-delay systems," Automatica, vol. 45, pp. 2265-2271, 2009.

[42] L. Zhang and P. Shi, "Stability, $l_{2}$-gain and asynchronous $H_{\infty}$ control of discrete-time switched systems with average dwell time," IEEE Transactions on Automatic Control, vol. 54, no. 9, pp. 2192 2199, 2009.

[43] D. Xie, Q. Wang, and Y. Wu, "Average dwell-time approach to $L_{2}$ gain control synthesis of switched linear systems with time delay in detection of switching signal," IET Control Theory E Applications, vol. 3, no. 6, pp. 763-771, 2009.

[44] G. Zhai, B. Hu, K. Yasuda, and A. N. Michel, "Piecewise Lyapunov functions for switched systems with average dwell time," Asian Journal of Control, vol. 2, pp. 192-197, 2000.

[45] R. T. Rockafellar and R. J.-B. Wets, Variational Analysis, vol. 317, Springer, Berlin, Germany, 1998.

[46] K. Deimling, Nonlinear Functional Analysis, Springer, Berlin, Germany, 1985.

[47] P. Miller and A. Michel, Differential Equations, Academic, New York, NY, USA, 1982. 


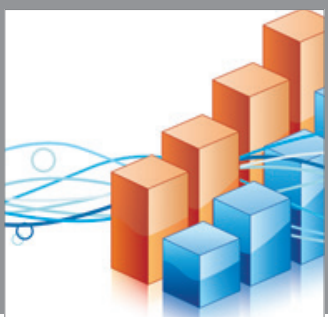

Advances in

Operations Research

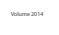

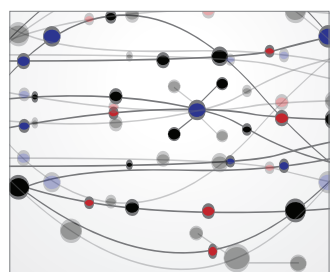

\section{The Scientific} World Journal
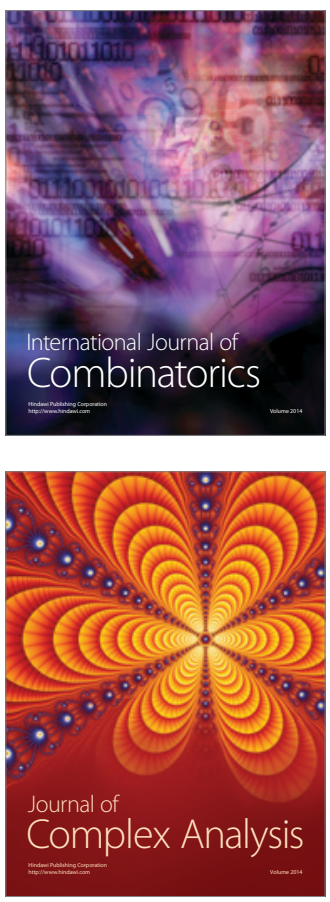

International Journal of

Mathematics and

Mathematical

Sciences
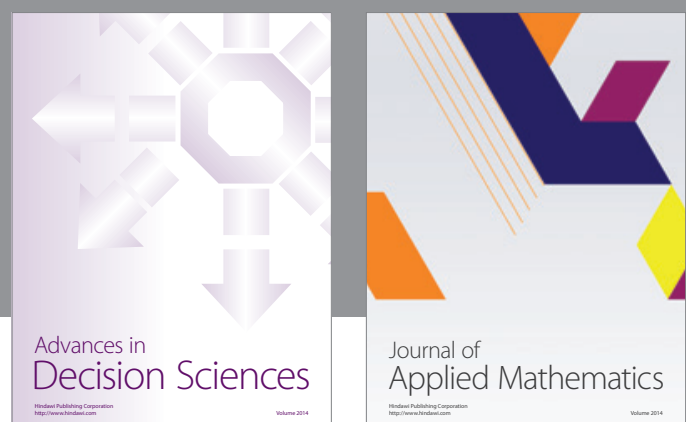

Journal of

Applied Mathematics
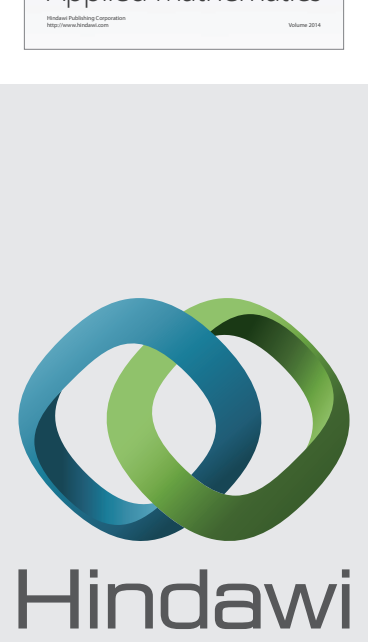

Submit your manuscripts at http://www.hindawi.com
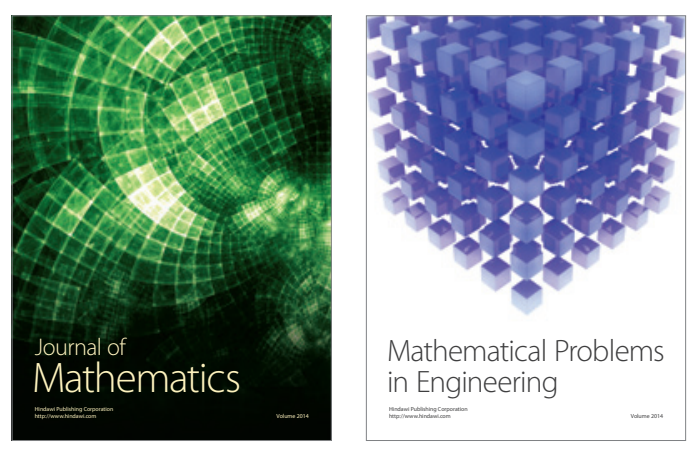

Mathematical Problems in Engineering
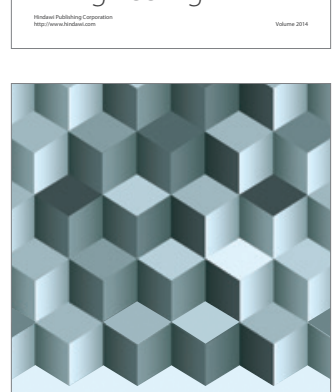

Journal of

Function Spaces
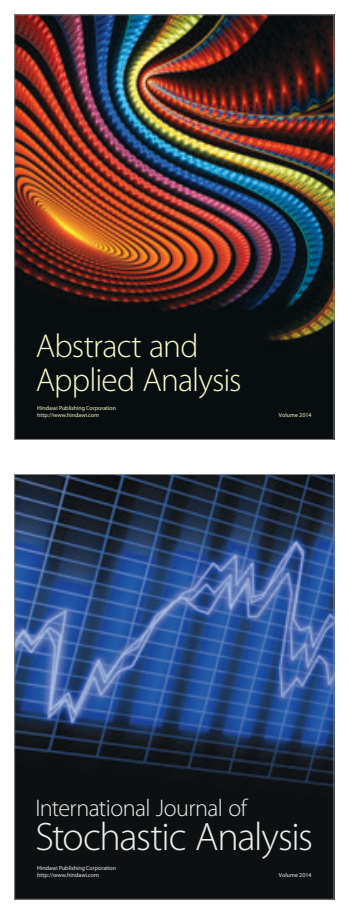

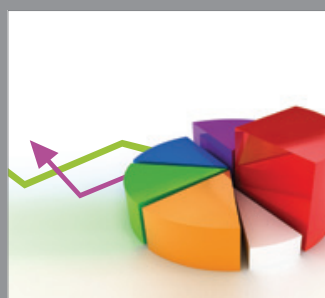

ournal of

Probability and Statistics

Promensencen
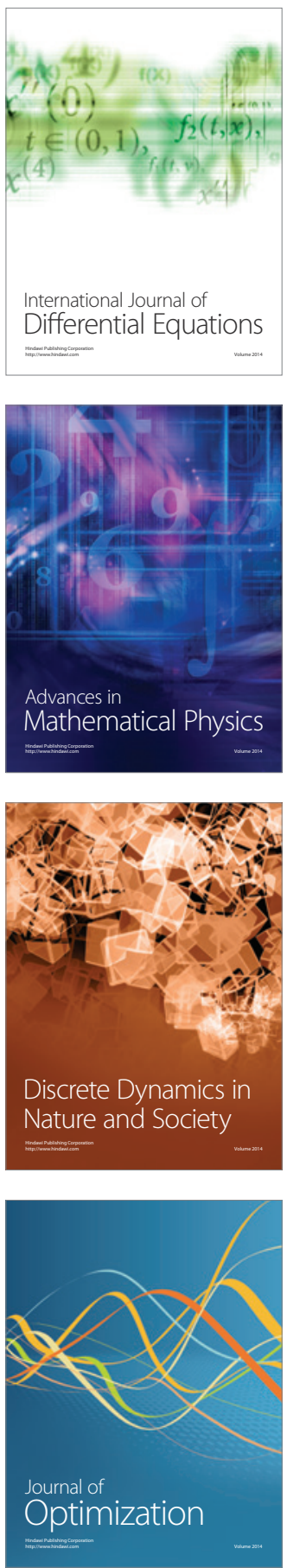